\title{
Radiative Feedback Effects during Cosmic Reionization
}

\author{
David Sullivan ${ }^{1}$ and Ilian T. Iliev ${ }^{1,2}$ \\ ${ }^{1}$ Astronomy Centre, Department of Physics \& Astronomy, Pevensey II Building, University of \\ Sussex, Falmer, Brighton BN1 9QH, United Kingdom \\ email: D.Sullivan@sussex.ac.uk \\ ${ }^{2}$ Speaker, email: I.T.Iliev@sussex.ac.uk
}

\begin{abstract}
We present coupled radiation hydrodynamical simulations of the epoch of reionization, aimed at probing self-feedback on galactic scales. Unlike previous works, which assume a (quasi) homogeneous UV background, we self-consistently evolve both the radiation field and the gas to model the impact of previously unresolved processes such as spectral hardening and self-shielding. We find that the characteristic halo mass with a gas fraction half the cosmic mean, $M_{c}(z)$, a quantity frequently used in semi-analytical models of galaxy formation, is significantly larger than previously assumed. While this results in an increased suppression of star formation in the early Universe, our results are consistent with the extrapolated stellar abundance matching models from Moster et al. 2013.
\end{abstract}

Keywords. Radiative transfer, methods: numerical, stars: formation, galaxies: formation, galaxies: high-redshift, intergalactic medium, large-scale structure of universe

\section{Introduction}

During the first billion years after the big bang, the large-scale cosmic web of structures we see today began to form. This was followed by the first stars and galaxies, which brought an end to the Dark Ages (Rees, 1999). These first luminous sources are thought to be the prime candidates which fuelled cosmic reionization, the last major phase transition of the Universe, from a neutral Inter-Galactic Medium (IGM) following recombination to the ionized state it remains in today. The physical processes which drive reionization encapsulate several areas of research, from cosmology and galaxy formation to radiative transfer and atomic physics. Even with the wealth of present-day observational information at our disposal, these processes are still not fully understood. Therefore we cannot model reionization analytically, instead turning to numerical simulations using observations to constrain our models.

Existing semi-analytical models of galaxy formation rely on Cosmological hydrodynamics simulations to calibrate their gas accretion recipes throughout cosmic time. To date, all such simulations have incorporated a (quasi) homogeneous, and instantaneous, UV background (e.g. Haardt and Madau, 2001; Faucher-Giguére et al., 2009) as a cheaper alternative to full radiation hydrodynamics (Shapiro et al., 1994; Gnedin, 2000a; Hoeft et al., 2006; Okamoto et al., 2008). Such models are however a crude approximation, neglecting much of the relevant physics during reionization (i.e self-shielding gas and spectral hardening). Photo-heating raises the temperature of the IGM to $T_{\text {IGM }} \sim 10^{4} K$, therefore the intergalactic Jeans mass increases substantially, raising the minimum mass of galaxies (Rees, 1986; Efstathiou, 1992; Gnedin and Ostriker, 1997; Miralda-Escude \& Rees, 1997). Halos whose virial temperature $T_{v i r} \lesssim T_{\text {IGM }}$ undergo photo evaporation due to their small potential wells, returning their gas to the IGM. This suppression at early 


\begin{tabular}{ccccc}
\hline Run & Box Size $(\mathrm{Mpc} / \mathrm{h})$ & Cells/Particles & Resolution $(\mathrm{kpc} / \mathrm{h})$ & Radiative Transfer \\
\hline ATON & 4 & $256^{3}$ & 15.6 & Yes \\
Fiducial & 4 & $256^{3}$ & 15.6 & No \\
\hline
\end{tabular}

Table 1. Summary of simulations. Radiative transfer/feedback was neglected in the fiducial model, allowing us to isolate the impact on gas during reionization.

times could be key to rectifying simulations with observations, addressing issues such as the missing satellites problem.

The primary motivation of this work is to improve existing semi-analytical models, by consistently following the evolution of radiation, gas, star formation and dark matter to probe this suppression. In $\S 2$ we briefly discuss the methods and codes to be used, followed by a description of the simulation parameters used. Finally, we present our results in $\S 3$ and offer some early conclusions and future work in $\S 4$.

\section{Codes and summary of simulations}

Despite the dawn of Petascale computing and large-scale parallel algorithms, modeling the complex interplay of both radiative and hydrodynamic feedback on the formation of the first stars and galaxies remains computationally challenging. Relevant time-scales differ by orders of magnitude between the gas and radiation, as the speed of light is much greater than the local sound speed, therefore fully-coupled radiation hydrodynamics (RHD) simulations require very short time steps to follow their evolution accurately. Initial attempts in the literature were limited to post-processing radiative transfer, however state-of-the-art algorithms have began to surface in recent years which perform this step in situ (Reynolds et al., 2009; Aubert and Teyssier, 2010; Wise \& Abel, 2010; Rosdahl et al., 2013 to list a few). We make use of the Eulerian Adaptive Mesh Refinement (AMR) code RAMSES (Teyssier, 2002) coupled to the ATON code (Aubert and Teyssier, 2008), which uses a moment-based radiative transfer scheme to follow the non-equilibrium thermochemistry of hydrogen.

RAMSES employs a second-order accurate hydrodynamics solver based on Godunov's method, a modern shock capturing scheme known for it's accuracy, and the N-body (dark matter and stars) using a Cloud In Cell (CIC) interpolation scheme on the mesh (Particle-Mesh, or PM). To accelerate the radiative transfer step the ATON code utilises graphical processing units (GPUs) to retain the full speed of light (up to 80x speed up over CPUs), however the complex coupling between GPUs and the conventional CPUs, which RAMSES uses, results in a loss of the AMR making the code uni-grid. To achieve galactic scale resolutions we have focused on small volume simulations here, however larger volume AMR simulations are currently underway (left to future discussion).

A brief summary of the setup for all simulations is presented in Table 1. Two simulations were carried out, with/without the radiative transfer of ionizing UV photons, initialised from redshift $z=100$ with identical initial conditions. A geometrically flat $\Lambda$ CDM Universe is assumed with cosmological parameters $\Omega_{m}=0.276, \Omega_{\Lambda}=0.724$, $\Omega_{b}=0.045, h \equiv H_{0} / 100 \mathrm{~km} \mathrm{~s}^{-1}=0.703$ and $\sigma_{8}=0.811$ where the symbols take their usual meanings. The star forming interstellar medium (ISM) is defined as all gas with over-densities $\Delta \equiv \rho /\langle\rho\rangle>50$. Both runs include supernovae feedback and were terminated at $z \sim 3$ due to time constraints.

Dark matter (sub) halos are catalogued using the Rockstar phase-space halo finder (Behroozi et al., 2013), adapted to work within the yt project (Turk et al., 2011). Particles are first divided into 3D Friends-of-Friends (FOF) groups which are each analysed in $6 \mathrm{D}$ phase space to give robust, grid and shape independent results. Those which are 


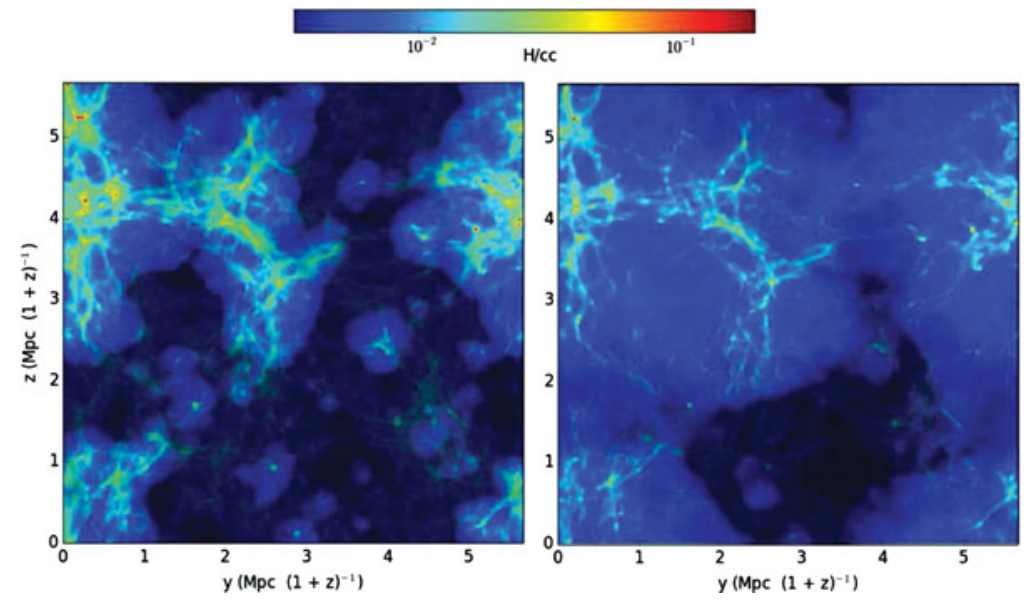

Figure 1. Full-box projections of the 3D volume for our ATON simulation. The colour bar denotes gas density at redshift 8.9 (left) and 7.5 (right) in units of hydrogen atoms per cubic cm. Dark/light regions correspond to neutral/ionized hydrogen, respectively. The box is completely reionized by $z \sim 6.5$, and demonstrates an inside-out reionization.

gravitationally unbound are removed and halo quantities are computed using the definition of the virial over-density from Bryan and Norman, 1998. Finally, merger trees are generated using the consistent-trees algorithm (Behroozi et al., 2013).

\section{Results}

The cosmic reionization of hydrogen can be characterised by two key stages, following the terminology of Gnedin, 2000b. During the initial "pre-overlap" stage, the Universe is predominately neutral with exception for small HII regions surrounding the first luminous sources. These ionization fronts initially propagate out slowly from high density filaments, until they reach the diffuse IGM. This occurs in approximately $10 \%$ of the Hubble time, leading to a sharp peak in the ionizing background and triggering an end to the "overlap" stage. There have been several studies which have investigated the progress and geometry of reionization (Ciardi et al., 2003; Iliev et al., 2006; Mellema et al., 2006; Zahn et al., 2007; McQuinn et al., 2007; Iliev et al., 2007; Alvarez and Abel, 2007; Mesinger \& Furlanetto, 2007; Geil and Wyithe, 2008; Choudhury et al., 2009), most of which are consistent with an inside-out progression, i.e where high density regions become ionized earlier, on average (Fig. 1).

Following the completion of the overlap stage, the gas must cool to enable collapse to high densities. In this regime atomic cooling is extremely efficient, as shown in Fig. 2 at $10^{4}<T<10^{5} \mathrm{~K}$ in both panels. Regions which were ionized later, predominately diffuse gas in the Circum-Galactic Medium (CGM) or voids, remain hotter on average due to their later, on average, reionization and longer cooling time $\left(t_{\text {cool }} \sim 1 / n_{H}\right)$, as well as some additional local heating due to shocks from supernovae and structure formation. In the fiducial case, most of the gas remains on the adiabat $T \propto \rho^{\gamma-1}$, where $\gamma$ is the ratio of specific heats, therefore the gas is cold enough to accrete onto low mass halos which are gas poor in our ATON simulation.

The temperature floor in the left panel of Fig. 2 acts to suppress gas accretion onto halos whose virial temperature is below that of the IGM. Pre-reionization, the gas fraction of halos scatters around the cosmic mean $\left\langle f_{b}\right\rangle \equiv \Omega_{b} / \Omega_{m}$ as a result of tidal interactions. Photo-heating leads to a sharp drop in the gas fraction of halos below a characteristic 

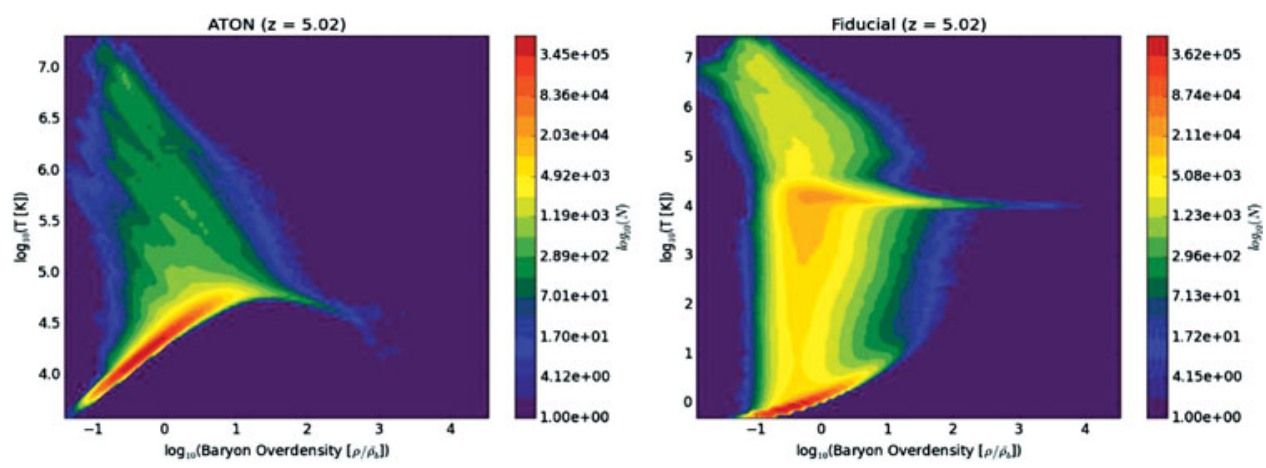

Figure 2. Phase diagrams in temperature-density space for our models. Gas at temperatures of $\sim 10^{4} \mathrm{~K}$ achieve equilibrium between photo-heating and atomic cooling, collapsing to high densities. Supernovae shock heat the gas to temperatures $\gg 10^{4} \mathrm{~K}$, which cannot be maintained by photo-heating alone. The color bar represents the number of cells occupying a given state.

mass scale $M_{c}(z)$, which sets the halo mass at which the gas fraction is half the cosmic mean. Low mass (mini) halos, whose potential wells are too small to retain photo-heated gas, are photo-evaporated resulting in a baryon mass of the order a fer percent of their total mass. Many semi-analytical models of galaxy formation implement the analytical formula proposed by Gnedin, 2000a to determine the gas fractions of halos:

$$
f_{b}(M, z)=\left\langle f_{b}\right\rangle\left\{1+\left(2^{\alpha / 3}-1\right)\left(\frac{M}{M_{c}(z)}\right)^{-\alpha}\right\}^{-\frac{3}{\alpha}}
$$

where $M$ is the halo mass, $M_{c}(z)$ is the characteristic mass and $\alpha$ is an exponent which controls how steep the transition is from baryon poor to rich. For $M \gg M_{c}(z)$ the first term dominates in the parenthesis, therefore the gas fraction of massive halos approaches the cosmic mean. Both Hoeft et al., 2006 and Okamoto et al., 2008 have tested this and found $\alpha=2$ gives good agreement with their simulations, albeit with varying conclusions on what sets $M_{c}$. Fig. 3 shows the redshift evolution of both the characteristic mass (right vertical axis) and $\alpha$ (left vertical axis), from Okamoto et al. Hoeft et al. and our ATON simulation. Radiative transfer leads to a significantly larger value for $M_{c}$ at all times in comparison to the two previous models, while $\alpha$ scatters between $\sim 1$ at early times to $\sim 2$ following reionization. Only distinct halos, identified from merger trees, are used here to remove contamination by tidal stripping.

Finally, we show stellar abundance matching models for both simulations listed in Table 1, with comparison to those of Moster et al., 2013, in Fig. 4. The shaded regions show the $1 \sigma$ deviation from the Moster et al. model, for which our ATON simulation shows very good agreement. Our fiducial model suffers from a high star forming efficiency from as early as $z=9$, suggesting the local reionization history is important prior to global reionization. Importantly, the agreement with Moster et al. is consistent at masses near the characteristic mass scale shown in Fig. 3, suggesting we are neither over or under suppressing star formation in these halos.

\section{Conclusions}

Using fully-coupled radiation hydrodynamics, we have probed the accuracy of semianalytical models of gas accretion during reionization commonly used in galaxy formation models. Radiative transfer is largely considered to be a second order component in most astrophysical applications, however the significance of the self-feedback as a result of such 


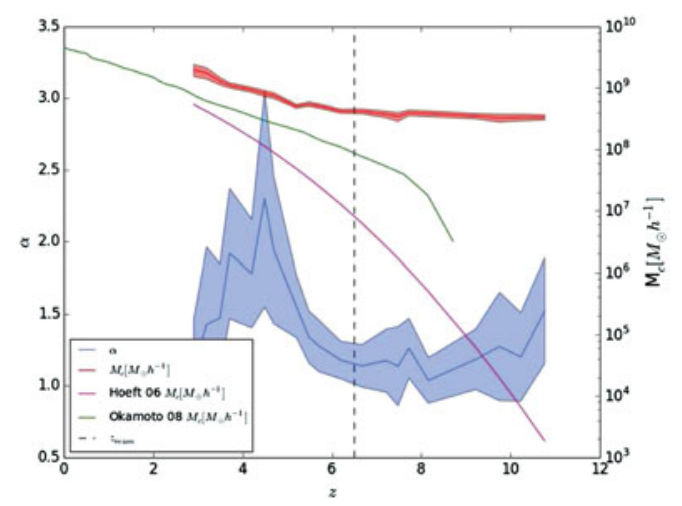

Figure 3. Evolution of the characteristic mass, $M_{c}$ (red) and the exponent $\alpha$ (blue) from Eqn. 3.1. The green and purple lines denote the values given by Okamoto et al., 2008 and Hoeft et al., 2006 , respectively. Shaded regions show $1 \sigma$ standard deviations, and the dashed line denotes the redshift of reionization for our ATON simulation.
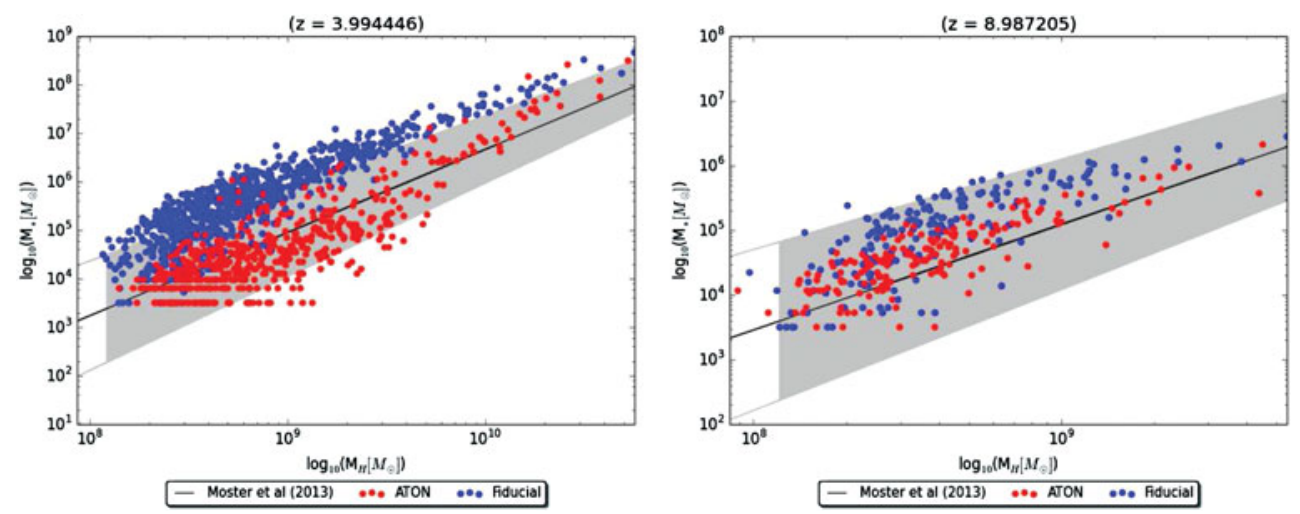

Figure 4. Stellar mass against halo mass with the $1 \sigma$ standard deviation (shaded) abundance matching model of Moster et al., 2013. The ATON simulation (red) shows strong agreement, suggesting that suppression during reionization is able to regulate early star formation in comparison to our Fiducial model (blue). It should however be noted that Moster et al. computed the relative abundance of stars from the Millennium simulation Springel et al., 2005 , which do not resolve halos below $\sim 10^{10} \mathrm{M}_{\odot}$, therefore the above is an extrapolation.

processes has been highlighted here. The increase in the characteristic mass shown in Fig. 3 is largely due to the inside-out progression of reionization in our simulations. Stellar sources of ionizing UV radiation form out of dense clumps, from which the first HII regions expand from. As the recombination time is large in such clumps, the majority of the soft-UV is absorbed, preferentially heating over-dense gas, with only hard-UV photons able to penetrate extending out to the surrounding diffuse gas. This effect is known as spectral hardening, which is absent from previous simulations (Hoeft et al., 2006; Okamoto et al., 2008) due to their (quasi) homogeneous UV background, hence these models under-predict the heating rate in over-dense regions. This was initially exposed by Okamoto et al., 2008, who artificially increased the heating rate as a function of density to test this hypothesis, arriving at the same conclusion as this work.

Our strong agreement with the stellar abundance match models of Moster et al., 2013 suggests that radiative feedback is very efficient at suppressing star formation during cosmic reionization, allowing baryons to remain in the IGM to fuel later periods of star formation. This is consistent with observations, which have long conflicted with 
numerical simulations which form stars too efficiently in the early Universe (TrujilloGomez et al., 2013 and references there-in). It is however not clear whether abundance matching models are accurate at the halo masses we have shown, and the results here are just an extrapolation. To probe whether our combination of radiative and supernovae feedback is feasible, larger simulation volumes must be explored to populate the rare high mass end of the halo mass function (we leave this to future work). Furthermore, our spatial resolution ( $\mathrm{dx} \sim 15 \mathrm{kpc} / \mathrm{h}$ comoving) is still quite coarse which may wash out much of the impact of self-shielding (see Fig. 2, no gas is resolved with densities $\rho / \overline{\rho_{b}} \gtrsim 10^{3}$ ). We will explore this with a series of upcoming simulations, using the AMR (RHD) code RAMSES-RT (Rosdahl et al., 2013).

\section{References}

Alvarez, M. a. \& Abel, T. (2007). MNRAS, 380(654).

Aubert, D. \& Teyssier, R. (2008). MNRAS, 387(1).

Aubert, D. \& Teyssier, R. (2010). ApJ, 724(1).

Behroozi, S., Wechsler, R., H., \& Wu, H. (2013). ApJ, 762(2).

Behroozi et al. (2013). ApJ, 763(1).

Bryan, G. L. \& Norman, M. L. (1998). ApJ, 495(1).

Choudhury, T. R., Haehnelt, M. G., \& Regan, J. (2009). MNRAS, 394(2).

Ciardi, B., Ferrara, A., \& White, S. D. M. (2003). MNRAS, 344(1).

Efstathiou, G. (1992). MNRAS, 256(2).

Faucher-Giguére, C.-A., Lidz, A., Zaldarriaga, M., \& Hernquist, L. (2009). ApJ, 703(2).

Geil, P. M. \& Wyithe, S. (2008). MNRAS, 386(3).

Gnedin, N. Y. (2000a). ApJ, 542(2).

Gnedin, N. Y. (2000b). ApJ, 535(2).

Gnedin, N. Y. \& Ostriker, J. P. (1997). ApJ, 486(2).

Haardt, F. \& Madau, P. (2001). Clust. Galaxies High Redshift Universe Obs. X-Rays, ed. D. M. Neumann J. T. T. Van (CEA Saclay).

Hoeft, M., Yepes, G., Gottl, S., \& Ring, C. (2006). MNRAS, 371(1).

Iliev, I. T., Mellema, G., Pen, U., Merz, H., Shapiro, P. R., \& Alvarez, M. A. (2006). MNRAS, $369(4)$.

Iliev, I. T., Mellema, G., Shapiro, P. R., \& Pen, U.-l. (2007). MNRAS, 376(2).

McQuinn, M., Lidz, A., Zahn, O., Dutta, S., \& Hernquist, L. (2007). MNRAS, 377(3).

Mellema, G., Iliev, I. T., Pen, U.-L., \& Shapiro, P. R. (2006). MNRAS, 372(2).

Mesinger, A. \& Furlanetto, S. (2007). ApJ, 669(2).

Miralda-Escude, J. \& Rees, M. J. (1997). ApJ, 497(1).

Moster, B. P., Naab, T., \& White, S. D. M. (2013). 20.

Okamoto, T., Gao, L., \& Theuns, T. (2008). 10.

Rees, M. J. (1986). MNRAS, 218(1).

Rees, M. J. (1999). Phys. Rep., 333.

Reynolds, D. R., Hayes, J. C., Paschos, P., \& Norman, M. L. (2009). J. Comput. Phys., 228(18).

Rosdahl, J., Blaizot, J., Aubert, D., Stranex, T., \& Teyssier, R. (2013). MNRAS, 436(3).

Shapiro, P. R., Giroux, M. L., \& Babul, A. (1994). ApJ, 427(1).

Springel et al. (2005). Nature, 435(7042).

Teyssier, R. (2002). A\&A, 385.

Trujillo-Gomez, S., Klypin, A., Col, P., Ceverino, D., Arraki, S., \& Primack, J. (2013). pre-print arXiv:1311.2910.

Turk, M. J., Smith, B. D., Oishi, J. S., Skory, S., Skillman, S. W., Abel, T., \& Norman, M. L. (2011). ApJ, 192(1).

Wise, J. H. \& Abel, T. (2010). 37.

Zahn, O., Lidz, A., McQuinn, M., Dutta, S., Hernquist, L., Zaldarriaga, M., \& Furlanetto, S. R. (2007). ApJ, 654(1). 